\title{
Adaptive Network Traffic Prediction Algorithm based on BP Neural Network
}

\author{
Ming Zhang ${ }^{1}$ and Yanhong $\mathrm{Lu}^{2}$ \\ ${ }^{1}$ Department of Electronic Engineering, Huaihai Institute of Technology; Lian \\ Yungang, China \\ ${ }^{2}$ Department of Mechanical and Electronic Engineering, Lianyungang Technical \\ College Lian Yungang, China \\ Lyg690916@163.Com
}

\begin{abstract}
With the rapid development of Internet technology, the network now has a large size and high complexity, and consequently the network management is becoming increasing difficult and complexity, so traffic forecast play a more and more role in network management. With a large amount of real traffic data collected from the actual network, an adaptive network traffic prediction algorithm based on BP neural network was proposed in this paper, it use an adaptive learning rate method to adjust the learning rate according to total error changing trend of decreased or increased and the difference of changing; and then it corrects the weights in each layers according to forward and reverse calculation. Simulation results show that, compared with the traditional BP neural network, our algorithm has better performance in the prediction results, and has smaller error.
\end{abstract}

Keywords: BP Neural Network, Network Traffic Prediction, Adaptive Learning Rate, Weight

\section{Introduction}

In recent years, with the rapid development of the Internet in the word and the rapid spread of various applications, Internet has become an integral part of people's daily work and life. In today's information society, due to the complexity of the network continues to increase and the new network businesses are emerging, along with the normal application of the internet, all kinds of abnormal traffic on the network has cropped affect normal internet running, threatening the hosts to safe and proper using. With the computer network size continuous expanding, network complexity continues increasing and the new network business continues appearing, the problem of understanding and analyzing the network traffic characteristics and behavior on internet has become increasingly prominent [1].

In order to obtain network traffic parameters and timely adjust the whole network configuration, the reasonable and urgent needs for a large network is to achieve intelligent control to greatly reducing the network workload of the administrator through measuring the network traffic and carrying out a detailed analysis or pumping sample analysis on each packet to reducing the various problems which may affect network QoS and security. Network preference monitoring is an integral part of network management, when a network failure, it can timely report and process, namely, it can timely understand the congestion and failure point to quickly adjust, according to the throughout, packet loss rate and end to end latency data and so on from the collected traffic information.

In this paper, an adaptive network traffic prediction algorithm based on BP neural network has been proposed, it use artificial neural networks technology to forecast the 
network traffic and then adjust the network traffic to improve network preference, achieve a normal and efficient operation of the network and make the network resources can be more fully and efficiently using.

\section{Related Work}

Network traffic prediction methods have been addressed by a number of researchers. Several well-known prediction models have challenged a few crucial problems. Grey model (GM) [2] will induce big residual errors around turning-point region in time series during the prediction. Due to the lack of a dynamic learning mechanism, Auto-regressive moving-average (ARMA) [3] cannot fit data sequences very well for irregular or nonperiodic time series.

When a feedback control system is closed via a communication channel, which might be shared with other nodes outside the control system, then called it as a networked control system (NCS) [4], it has many attractive advantages, such as, high system testability, resource utilization, low requirement to weight, space, power and wiring, it has widely used in process control [5], remote control [6], telephone-manipulation [7], robotics [8] and so on. Stability of networked control systems: explicit analysis of delay [9] discussed the influence of the sampling rate and network delay on system stability, and further studied the stability of NCS using a hybrid system stability analysis technique. Role of delays in networked control systems [10] indicated the impact of delay sources on the stability and performance of NCS, and showed a complete diagnostic profile of the role of delays in NCS. Real-time control systems with delays [11] analyzed NCS in discrete-time domain, and further modeled the network delay as constant, independently random, and random but governed by an underlying Markov chain. By solving a LQG optimal control problem, he generated a controller that guaranteed the system stability. Liu and Yao [12] used hidden Markov models to analyze NCS with delay governed by an underlying Markov chain with unknown probability distribution, and designed a stochastic optimal controller for NCS. Zhang et al., [13] also investigated in discrete-time domain the stabilization problem of NCS with random delay, and modeled the sensor-tocontroller delay and the controller-to-sensor delay as two Markov chains. They established the necessary and sufficient conditions on the existence of the stabilizing controllers, and used an iterative linear matrix inequality approach to calculate the statefeed back gains.

Artificial neural networks (ANNs) [14] started in the 1940s, it can model complex functional relationships according to utilize interconnected mathematical nodes or neurons to forma network. It has been evolved steadily and was adopted in many areas of science. It can help cognitive scientists to understand the complexity of the nervous system. Basically, the idea of artificial neural network is inspired by the learning process in the human brain. They are constructed and used as an alternative mathematical tool to solve the problem in the fields of system identification, prediction, pattern recognition, classification, process control, and many others [15]. Artificial neural networks have been widely used in the range of membrane technology applications [16]. However, there are several records in the literature which apply artificial neural network to predict SP in the electro-dialysis process.

One of the most received attentions is back-propagation neural network [17]. BPNs have hierarchical feed-forward network framework. In the classical structure of BPNS, in each layer, the outputs are directly sent to each neuron in the next layer. It has many layers, but at least there are three layers: one input layer is responsible for receiving and distributing input data, a hidden layer or intermediate layer is responsible for capturing the nonlinear relationship between inputs and outputs, and an output layer is responsible for producing the calculated data. BPNs also may contain a bias neuron to produce constant outputs, but it receives no input [18]. BP algorithm is based on minimization of 
errors in the neural network. The errors are described as the difference between the ideal outputs and the actual ones [19]. The training is completed when the precision of the training is met (Figure 1).

BPNs can be operated with better generalization and fault-tolerant capabilities, however, it has some short comings: Slow astringency can lead to a longer training time; local extreme point may emerge in the training process. So in literature [20], back propagation (BP) neural networks and improved BP algorithms were applied on the prediction of separation percent (SP), and their prediction capabilities could reflect generalization and adaptive abilities on complex data which had nonlinear relationships with each other. And with different structures of neural networks, transfer functions of neurons and learning rates, the optimum training parameters were obtained. Comparing BP neural networks with improved BP algorithms, improved BP algorithms were better than BP algorithm, due to changing with increasing ratios of learning rates and weights properly. And in the condition of high temperatures and voltages, the improved BP algorithms has better performance than BP neural networks, this was because improved BP algorithms had the generalization ability for high values.

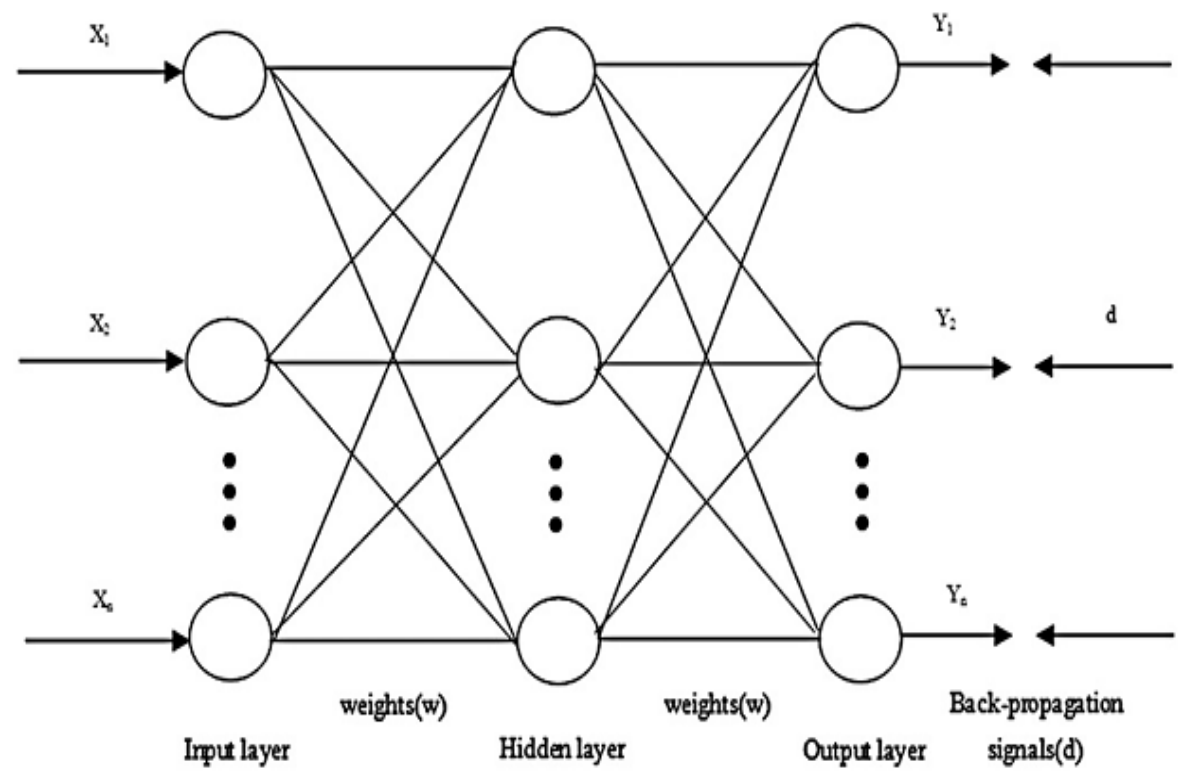

Figure 1. The Structure of BPNS

Since the appropriate parameters (weight) were chosen after training, backpropagation artificial neural network (BPANN) [21] may have trouble with the problem of over-fitting or under-fitting result. Adaptive neuron-fuzzy inference system (ANFIS) [22] model cannot avoid volatility clustering problem [23], it is easy to lead to a lot of bad accuracy in non-periodic short-term. Notably ANFIS can possibly overcome the problem of over fitting and automatically adjust the training results by itself. Combined the nonlinear generalized auto regressive conditional heterogeneity (NGARCH) model with ANFIS system was proposed to tackle the overshoot and volatility clustering effects at the same time during single-step-lookahead prediction. A novel hybrid approach, combining adaptive neuron-fuzzy inference system (ANFIS) with nonlinear generalized auto regressive conditional hetero-scedasticity (NGARCH) was proposed in [24], it was tuned optimally by quantum minimization (QM) and then applied to forecasting the flow of data packets around website. The composite model (QM-ANFIS/NGARCH) is setup in the forecast point of view to improve the predictive accuracy because it can resolve 
the problems of the over-shoot and volatility clustering simultaneously with in time series. As part of real-time intelligence web analytics, the high accurate prediction will aid webmaster to improve the throughput of data-packet-flow up to around 20\%, with helping each webmaster to optimize their website, maximize on line marketing conversions, and lead campaign tracking.

\section{Adaptive Network Traffic Prediction Method based on BP Neural Network}

BP neural network can use any differentiable function as its transfer function. Stype function, tangent S-type function and linear functions are commonly functions. Using different transfer functions will achieve different output values. Using a linear function of time, it can get the output value of any size.

\subsection{Network Traffic Measurement Method}

Usually, there are three measurement method of network traffic:

(1) Using SNMP to query network service, such as RMON;

(2) Monitoring packet-based methods, such as Sniffer products;

(3) Using IP FLOW techniques, such as Netflow.

The first method can get information of network traffic size, such as number of packets about input/ output through network interface and number of bytes, but it can not provide the detailed information about application type in network traffic; the second method can provide highly accurate information about network traffic based on packet level, but it is difficult to implement in high-speed links because of the high amount of data acquisition; the third method can provide detailed information about the applications type of network traffic and the amount of collected data is less than the second method. So it can be seen that the method which using IP Flow to measure and analysis network traffic is a more effective and compromise method. Netflow is a method of data exchange, it uses the standard switching method to process the first IP packet of data stream and generate Netflow cache, then the same data based on cache information is transmits at the same data stream and it is not matches the associated access control policy, at the same time, Netflow contains the statistics information of data stream.

There are four parts: traffic data generation, data collection, filtering of data traffic, analysis and process of abnormal flow.

(1) Traffic data generation: Netflow data was generated through Cisco routing. We set the data collection server ( IP address: 210.28.39.88)to listen Netflow data on port 9995 in real time, so it can start the process of receiving data when there are Netflow data.

(2) Data collection: when data sent over, acquisition sever will obtain the Netflow data which ensures the packets is not dropping as much as possible, then data collection module process the Netflow raw data in the log file to measure network traffic.

(3) Filtering of data traffic: when traffic acquisition is completed, it uses the function of filtering, screening, arrangement and so on in Flow-tools software to classify the collection data. It can be classified separately in a variety of applications, it can also be sorted according to size of the data flow, so the network-administrators can facilitate observe the data and find out abnormal traffic. Flow-filter can get data traffic through a specific port or protocol by filtering the collected data, can understand network traffic according to demanded needs, it can be combined with other tools to draw flow chart to analysis the network performance and operation.

(4) Analysis and process of abnormal flow: it can get the main performance in network through statistics and computing the collected data flow and form regularly reports and maintain the database of network traffic or log of network traffic. Under abnormal 
circumstances, it uses neural network to analysis abnormal flow and effectively determine these situation is a malicious attack which threaten network security or some special network usage. Neural networks have a strong self-learning ability, it can early enough to find new viruses and intended attacking before the virus and firewall updated, and it can truncate data flow which threaten network security in a timely and effectively protect network security to minimize the loss.

Neural network model using BP model, it uses three layers form of the neural network structures, the input neuron layer, hidden neurons layer and output neurons layer. BP learning algorithm is a back-propagation method. The algorithm uses the mean square error and gradient descent algorithm to achieve the correction of the network connection weights, and its goal is to minimize the difference between mean square error of the actual output and regulations output.

After the network traffic is abnormal, the feature vectors will be extracted which can be completely and accurately represent data flow according to analysis and process for these abnormal data flow, and then submit these feature vector to the neural network engine as the neural network input. Neural network engine analysis and process this feature vector to determine whether the behavior is an intrusion, if it is an attack, then it will send a warning message to the user and record this information in the log file. If the alarm information for an attacker to improve and update the sample library has great value, for example, found a new type of aggressive behavior, you can add the alarm in the attack sample library to prepare for further-learning, thereby it can increase the processing capacity for neural network. Figure 2 is the principle of neural network:

We first obtain a sample of typical network attacks as a learning sample database and use these samples to train the neural network classification engine. After subsequent training, the neural network store these characteristic pattern of aggressive behavior in the form of weights, so when the neural network really run, it can analysis and process these captured network data flow, and to determine which is the normal or malicious. If a new and unknown attack was found, it will be fed back to the training sample in the library. This reflects the neural network classification engine with the ability of self-learning and processing unknown attacks.

\subsection{Back-Propagation (BP) Neural Network Model}

A typical BP neural network model is a full-connected neural network including input layer, hidden layer and output layer [25].The goal of the training process is used to find the weights that minimize some overall error measures, such as the sum of squared errors (SSE) or mean squared errors (MSE).Hence the network training is actually an unconstrained nonlinear minimization problem. 


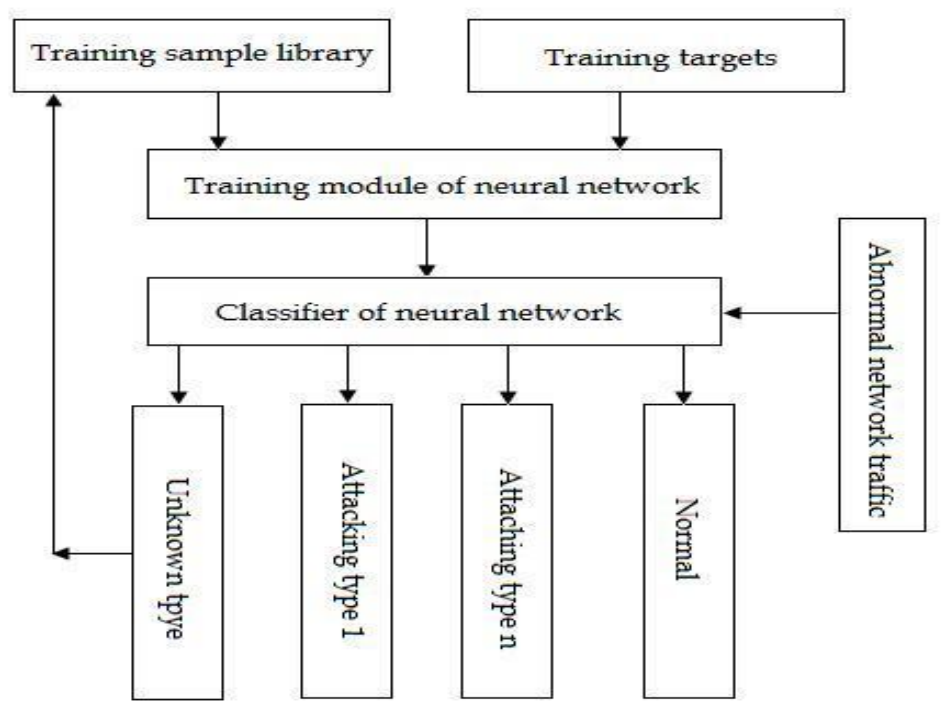

Figure 2. Working Principle of Neural Network Engine

For the prediction of network traffic, the sequence numbers of network traffic is associated with a multi-step, namely, there is a certain relationship between the current sample flow and the past sampling period. Assuming that $x_{k}$ $(\mathrm{k}=1,2, \ldots \mathrm{N})$ denotes the traffic of the kth sampling interval, the flow forecasting model of the relevant step $\mathrm{p}(\mathrm{p} \geq 1)$, ahead step $1(1 \geq 1)$ is defined as:

$$
x_{k+1}=f\left(x_{k}, x_{k-1}, \ldots x_{k-p+1},\right)
$$

Namely, $\mathrm{f}$ is the relationship between the $\mathrm{k}+\mathrm{lth}$ flow of the $\mathrm{k}+\mathrm{l}$ th sampling interval and the $\mathrm{p}$ number of flow before it, $\mathrm{f}$ is a nonlinear function which is difficult to explicitly described, BP neural network is more suitable to solve this problem. BP neural network traffic prediction model is shown as follow:

In Figure 3, the sensor, the network, and the Delay predictor using a BP neural network make up a virtual sensor. The virtual sensor adopts variable sampling period, which equals to the predicted delay obtained from the delay predictor. If we only watch the whole virtual sensor box from its outside and do not consider its internal structure, the NCS is then turned into an instant sampling system, i.e., there is no time delay. This is consistent with the variable-period sampling model. Within the virtual sensor box, we make the time delay prediction for the sampling period of the NCS to dispel the influence of the time delay [4].

Figure 4 shows that the prediction structure of BP neural network, namely, it has input layer, output layer and hidden layer. The input layer has $\mathrm{p}$ neurons, it received $\mathrm{p}$ difference traffic as input, namely, the inputs are $x_{k}, x_{k-1}, \ldots x_{k-p+1}, \mathrm{p}$ is the number of steps between the predicted traffic flow a $x_{k+1}$ nd the its previous traffic flow. Hidden layer has n neurons, output layer has one neuron which its output $\overline{x_{k+l}}$ is the prediction of flow difference of the $\mathrm{k}+\mathrm{l}$ th $[26], w_{i j}^{m}$ is the connection weights between the neuron $\mathrm{j}$ in m-1 layer and m layer. $a_{i k}^{m}$ is the $\mathrm{k}$ th input of neuron $\mathrm{i}$ in the mth layer, $g_{m}$ is the transfer function, $b_{i k}^{m}$ is the corresponding output. The specific formulas are as follows. 


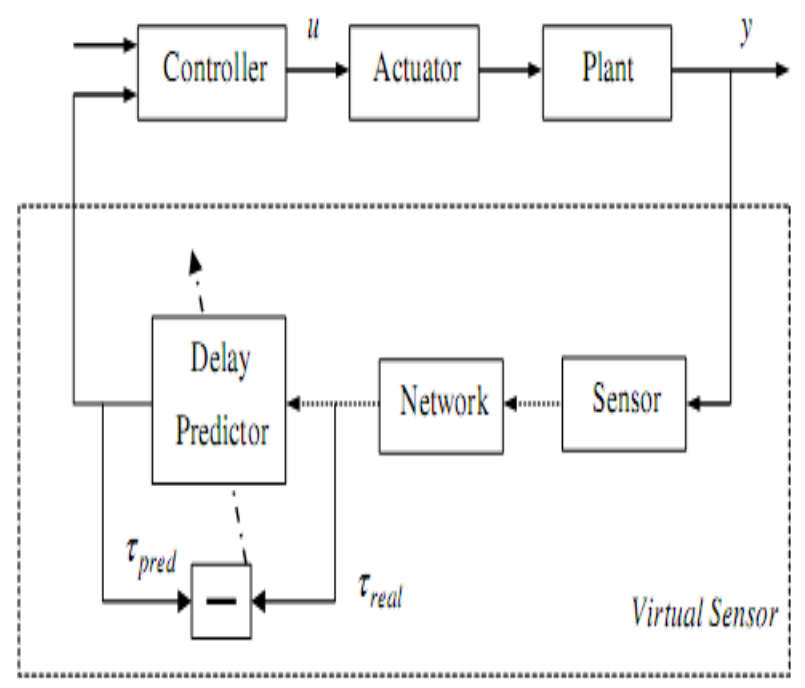

Figure 3. Configuration of the Networked Control System with Delay Prediction

$$
\begin{array}{r}
a_{i k}^{m}=\sum_{j} w_{i j}^{m} b_{j k}^{m-1} \\
b_{i k}^{m}=g_{m}\left(a_{i k}^{m}\right) \\
b_{i k}^{1}=x_{k-i+1}, b_{i k}^{3}=\overline{x_{k+1}}
\end{array}
$$

In the $k+1$ th sampling interval, the $x(k)$ is known, namely, $x(k)=\left(x_{k}, x_{k-1}, \ldots x_{k-p+1},\right)$, the $x_{k+1}$ can be computed according to the input $\mathrm{x}(\mathrm{k})$.

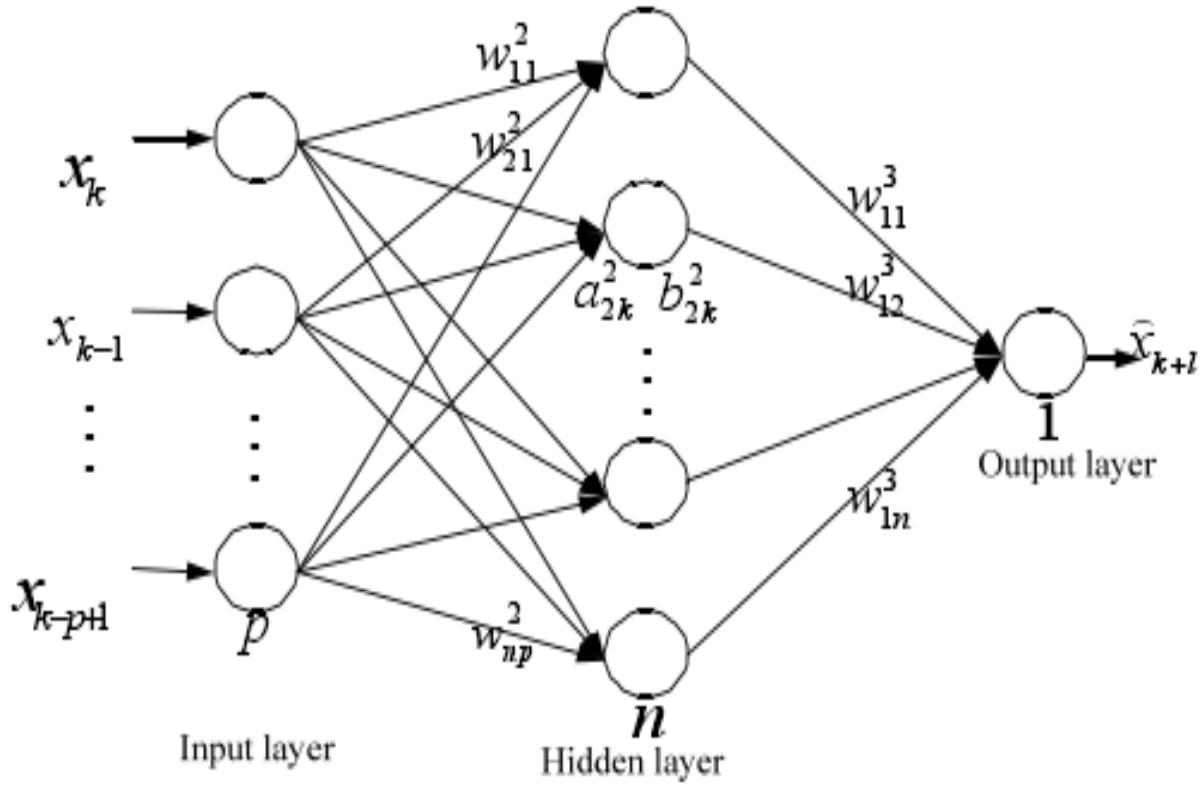

Figure 4. The Prediction Model of BP Neural Network 


\subsection{Training Algorithm of Weights in Layers}

Since the initial value of connection weights in the neural network layers are arbitrary, the neural network must be trained to assure that the deviation between the desired output value and the actual value is as small as possible, BP neural network reversely send the error between the real output of sample to neurons in layers, it constantly adjusts the weights of layers using the gradient descent method to reduce the error caused by the weight, so it can assure that the error between the real output of training samples and the output of neural network limits in a set range or the number of training is maximum.

When the network output is $\overline{x_{k+1}}$, the error $E_{k}$ is as follows:

$$
E_{k}=\frac{1}{2}\left(x_{k+1}-\overline{x_{k+1}}\right)^{2}
$$

The total error $\mathrm{E}$ is

$$
E=\sum_{k=1}^{n} E_{k}
$$

Where $\mathrm{n}$ is the number of samples.

Adjust weights. Using gradient descent method to solve the minimum of error function, the variable quantity of $\omega_{i j} \Delta\left(\omega_{i j}\right)$ was expressed as follows:

$$
\Delta \omega_{i j}=-\frac{\partial E}{\partial \omega_{i j}}
$$

$\eta$ was learning late.

\subsection{Improved BP Algorithm}

There were some methods about improved BP algorithms, such as, additional momentum method, adaptive learning rate method, flexible BP algorithm and so on. In this paper, adaptive learning rate method and improved training algorithm for each layers were used to predict separation percent and compared prediction capability with that of BPNs [20].

\section{(1) Adaptive learning rate method}

The basic principle of adaptive learning rate: when learning rate $(\eta)$ was increased, this caused shortening learning time; the higher the learning rate, the harder the convergence, and in this condition, learning rate should be decreased until the convergence of the training process. Learning rate was adjusted by the changing of error sand gradients, and also by the gradients of the learning rate according to error function. More over, the changing of the total error may be proceeded by adjusting heuristically, the rules were as follows:

a. If the total error (E) decreased and the difference of changing is bigger than $\tau \Delta \omega_{i j}$, learning rate needed to increase; else the learning rate is constant.

b. If the total error (E) increased and the difference of changing is bigger than $\tau \Delta \omega_{i j}$, learning rate needed to decrease.

The specific formula was as follows: 


$$
\eta(k+1)= \begin{cases}\gamma_{1} \eta(k) & \mathrm{E}\left[\mathrm{x}_{k+1}\right]<\mathrm{E}\left[\mathrm{x}_{k}\right] \text { and } \mathrm{d}>\Delta \tau \\ \gamma_{2} \eta(k) & \mathrm{E}\left[\mathrm{x}_{k+1}\right] \geq \mathrm{E}\left[\mathrm{x}_{k}\right] \text { and } \mathrm{d}>\Delta \tau \\ \eta(k) & \text { ot her } \mathrm{s}\end{cases}
$$

Where $d$ is the absolute difference of $E\left(x_{k+1}\right)$ and $E\left(x_{k}\right), \eta$ is the learning rate; $k$ is the training times; $\mathrm{E}$ is error function as formula (6) shows;

(2) Data preprocessing

All the training data are rescaled to a specific range for a time series forecasting problems. When the extreme values of discharge are utilized, the gradient of transfer function will approximate to zero, consequently, leading to slow the training down [27]. To overcome the problem of extreme events some studies suggest rescaling the data into a smaller interval of $[0.1,0.85]$ (or[0.1,0.9]) [28]. Here we apply an experimental data preprocessing method [29], which can rescale all the training data to the range of [0.1,0.9] [30]. The formula can be written as

$$
\bar{x}_{i}=\frac{0.1\left(x_{i}-x_{\max }\right)+0.9\left(x_{\min }-x_{i}\right)}{x_{\min }-x_{\max }}
$$

Where $x_{i}$ is the observed value, $x_{\max }$ and $x_{\min }$ are respectively denote the minimum and maximum value of data series.

(3) Improved training algorithm

The more detailed improved training algorithm are described as follows:

Step 1: Initialize the weights $w_{i j}^{m}$ of BP neural network layers.

Step 2: Use training sample to train BP neural network. Repeat the following process until meeting the requirements error.

First: Forward calculation: calculate the $a_{i k}^{m}, b_{i k}^{m-1}$ and $\bar{x}$ of neuron in each layer.

Reverse calculation process: calculate $\delta_{i k}^{m}$ of each neuron in m layer.

Second: Use the following formula to correct weights

$$
w_{i j}^{m}=w_{i j}^{m}+\eta(k+1) \frac{\partial E_{k}}{\partial w_{i j}^{m}}
$$

Where $\eta(k+1)$ is the learning rate which can be calculate by formula (8), and it can be dynamic adjust for each $\mathrm{k}$ according to the error margin and changing trends

Step 3: $\left(x_{k}, x_{k-1}, \ldots x_{k-p+1}\right)$ is the input of BP neural network, then the output is the prediction of flow difference of the $\mathrm{k}+\mathrm{l}$ th.

Step 4: The subscript of samples are moving one step forward, go to step 2 to continue to the next forecast.

\section{Simulation Results}

This paper selects the flow library:http://oa.hhit.edu.cn/general/2010, the time series of network flows in master node router from 10 January 2010 to 9 July which has 180 days is the testing sample for BP neural network and normalization.

\subsection{Performance Computation}

In order to test the effect of prediction, it uses some indicators, such as MAE (Mean Absolute Error), MSE (Mean Square Error), SSE (Sum Square Error), NMSE (Normalized Mean Square Error) et al to test prediction performance. 


$$
\begin{array}{r}
M A E=\frac{1}{n} \sum_{k=1}^{n}\left|x_{k}-\overline{x_{k}}\right| \\
M S E=\frac{1}{n} \sum_{k=1}^{n}\left(x_{k}-\overline{x_{k}}\right)^{2} \\
N M S E=\frac{1}{\sigma^{2}} \frac{1}{n} \sum_{k=1}^{n}\left(x_{k}-\overline{x_{k}}\right)^{2}, \sigma^{2}=\frac{1}{n} \sum_{k=1}^{n}\left(x_{k}-\bar{x}\right)^{2} \\
S S E=\sum_{k=1}^{n}\left(x_{k}-\bar{x}_{k}\right)^{2}
\end{array}
$$

Obviously, the forecasting accuracy is higher as smaller as the value of indicators .

\subsection{Computation of Prediction Value with Actual Value}

In order to compare the error of prediction, at the same conditions, respectively running 10 times for tradition BP neural network and improved BP neural network, and assume

\begin{tabular}{|c|c|c|c|c|}
\hline Experiment & algorithm & MSE & MAE & SSE \\
\hline 1 & $\begin{array}{l}\text { Traditional } \\
\text { algorithm }\end{array}$ & $\begin{array}{ll} & 0.016 \\
3 & \end{array}$ & $\begin{array}{ll} & 0.197 \\
3 & \\
\end{array}$ & $\begin{array}{ll} & 0.326 \\
1 & \end{array}$ \\
\hline 2 & Adaptive algorithm & $8 \begin{array}{l}0.003 \\
\end{array}$ & $1 \begin{array}{l}0.052 \\
\end{array}$ & $2 \quad 0.076$ \\
\hline
\end{tabular}
that the error is 0.0001 in the experiment. The average of prediction value in 10 times is tests the stability.

Table 1. The Prediction Performance of Two Algorithms

After completion of the experiment, the prediction preference of network traffic data for two type algorithms as shown in Table 1, wherein, MSE is the mean square error, SSE is the sum squared error, MAE is the mean absolute error, NMSE is the normalized mean square error. Experiment 1 uses tradition BP neural network algorithm to forecast the network flow, the predicted test curves are shown in Figure 5. Experiment 2 uses improved BP neural network algorithm to forecast the network flow, the predicted test curves are shown in Figure 6, wherein the solid line represents the actual value of the network traffic and the dashed line represents the predicted value.

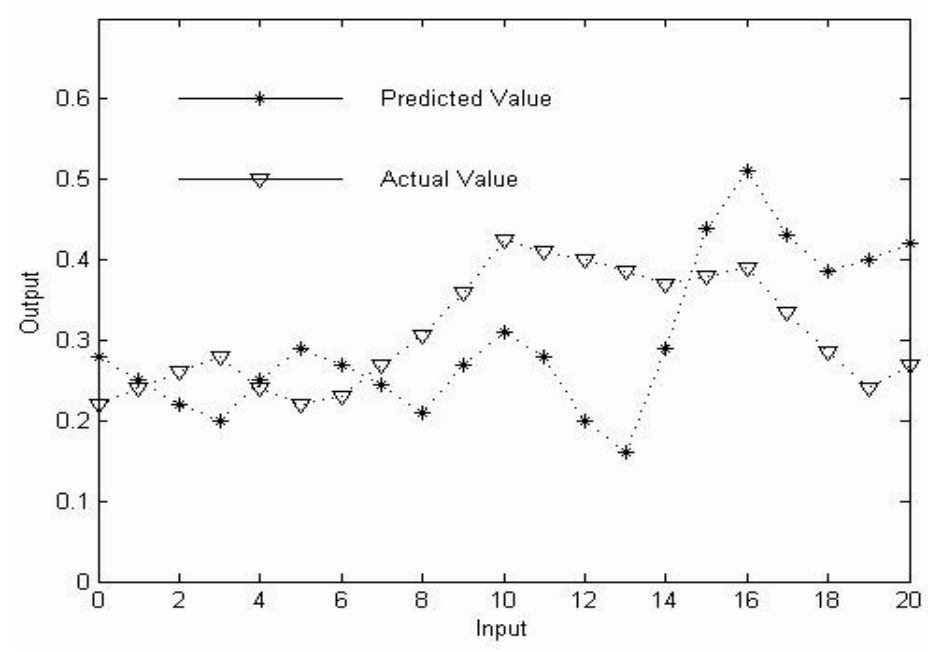

Figure 5. The Prediction Graph of Traditional BP Neural Network 
The results can be seen from Table 1, the improved BP neuron network algorithm has better performance in network traffic prediction. Wherein, if the MSE and SSE are smaller, it denotes that it has higher fit the network traffic; if the MAE is lower, it means that the prediction effect of network model is better. The maximum relative error can be expressed the risk level of the forecasting methods. The experimental results in Table 1 denote that the improved BP neural network algorithm has the maximum MRE, means that it has more stable and reliable than the tradition BP neural network algorithm. Test results from Figure 5 and Figure 6 can reflect the trend of two algorithms, but the forecast of the improved BP neural network algorithm is more accurate.

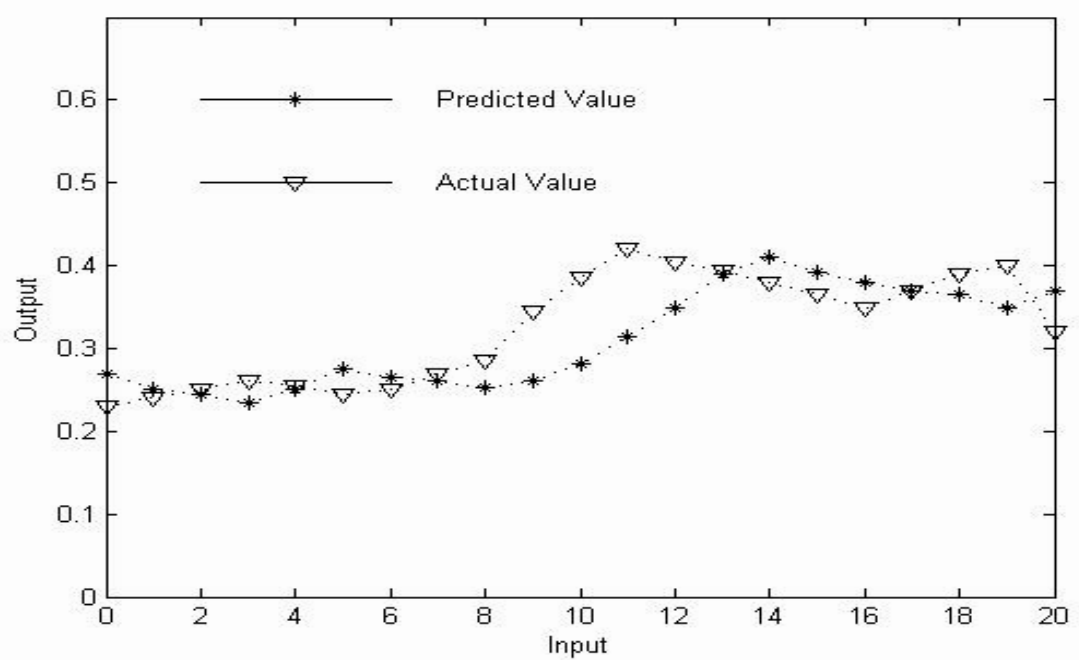

Figure 6. The Prediction Graph of Improved BP Neural Network

\section{Acknowledgements}

We acknowledge the support of National Natural Science Foundation of China (61174013), National Development and Reform Commission foundation of China (2013-1309-71), Six Personnel Peak Project of Jiangsu Province (DZXX-055) and Industrial Research Projects of Lianyungang (CG1309), we sincerely thank the anonymous reviewers for their constructive comments and suggestions.

\section{References}

[1] H. Lei and C. Perkins, "Ad HOC Networking with Mobile IP[C]//Proceedings Of The Second European Personal Mobile Communications Conference”, Bonn, Germany, September 30-October 2, 1997:197202.

[2] B.R. Chang, "Hybrid BPNN-Weighted Grey-CLMS Forecasting", Journal Of Information Science And Engineering, 21(1), 2005:209-221.

[3] G.E.P. Box, "G.M.Jenkins,G.C.Reinsel," Time Series Analysis: Forecasting \& Control, Prenticehall,Newjersey, 1994.

[4] J.Q. Yi, "BP Neural Network Prediction-Based Variable-Period Sampling Approach For Networked Control Systems", Applied Mathematics And Computation, 185, 2007: 976-988.

[5] S.Yang, X.Chen and J.L. Alty, "Design Issues and Implementation Of Internet-Based Process Control Systems", Control Engineering Practice, 11, 2003: 709-720.

[6] K.C. Lee, S. Lee, "Remote Controller Design Of Networked Control Systems Using Genetic Algorithm", Proceedings Of IEEE International Symposium On Industrial Electronics, 3, 2001: 1845-1850.

[7] T.T. Ho and H. Zhang, "Internet-Based Tele-Manipulation", Proceedings Of IEEE Canadian Conference On Electrical And Computer Engineering, 3, 1999: 1425-1430.

[8] K. Han, S.Kim, Y.Kim and J.Kim, "Internet Control Architecture For Internet-Based Personal Robot", Autonomous Robots, 10, 2001: 135-147.

[9] M.S. Branicky, S.M. Phillips and Weizhang, "Stability of Networked Control Systems: Explicit Analysis of Delay", Proceedings of American Control Conference, 4, 2000: 2352-2357. 
[10] M.S. Mahmoud and A. Ismail, "Role of Delays in Networked Control Systems", Proceedings Of 10th IEEE International Conference On Electronics, Circuitsand Systems, 1, 2003: 40-43.

[11] J.Nilsson, "Real Time Control Systems With Delays", Ph. D. Dissertation Of Dept. Automatic Control Of Lund Institute Of Technology, Sweden,1998.

[12] F. Liu and Y. Yao, "Modeling and Analysis Of Networked Control Systems Using Hidden Markov Models", Proceedings Of International Conference On Machine Learning And Cybernetics, 2, 2005: 928-931.

[13] L. Zhang, Y. Shi, T. Chen and B. Huang, "A New Method For Stabilization Of Networked Control Systems With Random Delays", Proceedings Of American Control Conference, 1, 2005: 633-637.

[14] W. Sha and K. L. Edwards, "The Use Of Artificial Neural Networks In Materials Science Based Research", Mater. Des. 28, 2007: 1747-1752.

[15] F. S. Mjalli, S. Al-Asheh and H. E. Alfadala, "Use Of Artificial Neural Network Black-Box Modeling For The Prediction Of Waste Water Treatment Plants Performance", J. Environ. Manage. 83, 2007: 329338.

[16] M. Sadrzadeh, T. Mohammadi, J. Ivakpour and N. Kasiri, "Separations Of Lead Ions From Waste Water Using Electro-Dialysis: Comparing Mathematical And Neural Network Modeling”, Chem. Eng. J. 144, 2008: 431-441.

[17] B. H. M. Sadeghi, “A BP-Neural Network Predictor Model for Plastic Injection Molding Process”, J. Mater. Process. Technol.103, 2000: 411-416.

[18] J. A. Anderson, “An Introduction to Neural Networks", MIT Press, Cambridge, MA, 1995.

[19] B. Irie and S. Miyake, "Capabilities Of Three-Layered Perceptron", Proceedings Of The 2nd IEEE International Conference On Neural Networks, San Diego, California, USA, 1, 1988: 641-648.

[20] G.L. Jing, W. Du and Y.Guo, "Studies On Prediction of Separation Percent in Electro-Dialysis Process via BP Neural Networks and Improved BP Algorithms", Desalination, 291, 2012: 78-93.

[21] S. Haykin, "Neural Networks", 2nd Edition, Prentice Hall, New Jersey, 1999.

[22] J. S. R. Jang, “ANFIS: Adaptive-Network-Based Fuzzy Inference Systems”, IEEE Transactions On Systems, Man, And Cybernetics, 23(3), 1993: 665-685.

[23] C. Gourieroux and A. R. C. H. Models, "Financial Applications", Springer-Verlag, New York, 1997.

[24] H.F. Tsai, B.R. Chang, "Novel Hybrid Approach To Data-Packet-Flow Prediction For Improving Network Traffic Analysis", Applied Soft Computing, 9, 2009: 1177-118.

[25] ASCE Task Committeeon Application Of Artificial Neural Networks In Hydrology, "Artificial Neural Networks In Hydrology I: Preliminary Concepts”, Journal Of Hydrologic Engineering, 5, 2000: 115-123.

[26] C. Zhen-We, I.G. Zheng-Wei, "Simulation Realization Of Prediction Model Based On Wavelet Neural Network", Computer Simulation, 25(6), 2008:147-150.

[27] C. W. Dawson and R. L. Wilby, "Hydrological Modeling Using Artificial Neural Networks", Progress In Physical Geography, 25, 2001: 80-108.

[28] K. C. Luk, J. E. Ball and A. Sharma, "A Study Of Optimal Model Lag And Spatial Inputs To Artificial Neural Networks For Rainfall Forecasting”, Journal Of Hydrology, 227, 2000: 56-65.

[29] D. Srinivasan, A. C. Liew and C. S. Chang, "A Neural Network Short-Term Load Forecaster", Electric Power Systems Research, 28, 1994: 227-234.

[30] Q. Ju, Z. Yu, Z. Hao, G. Ou, J. Zhao and D. Liu, "Division-Based Rainfall-Runoff Simulations With BP Neural Networks And Xinanjiang Model”, Neuron Computer, 12, 2008: 1-10

\section{Authors}

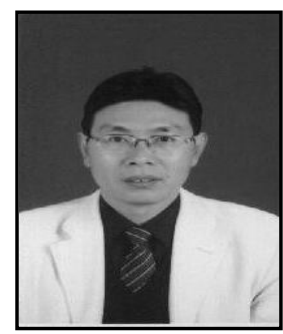

Ming Zhang, is an professor with the School of Electronic Engineering, Huaihai Institute of Technology, Lian yungang,China.He received his doctor degree in Information Network from Nanjing University of Posts \& Telecommunications, Nanjing, China in 2011. His current research interests include wireless sensor networks, network control and software technology.

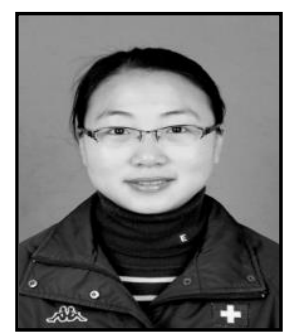

YanHong Lu, is a $\mathrm{n}$ associate professor with Department of Mechanical and Electronic Engineering, Lianyungang Technical College, Lian yungang, China. She received her M.S. from East China Normal University in 2010. Her current research interests include wireless sensor networks, network control and software technology. 